\title{
Flegmasia cerulea dolens. Reporte de casos
}

\author{
Phlegmasia cerulea dolens. Cases report
}

Oswaldo Mateo Silva Rodríguez ${ }^{1, a}$

\section{RESUMEN}

Laflegmasia cerulea dolens (FCD, flebitis, cianosis, dolor) es una complicación rara de la trombosis venosa profunda, se debe a obstrucción del retorno venoso, generalmente del miembro inferior, con cianosis severa y compromiso circulatorio que compromete vasos colaterales, a diferencia de la flegmasía alba dolens que no compromete las colaterales. El cuadro clínico se caracteriza por dolor de inicio súbito, debido al aumento significativo de la presión venosa, que lleva a ausencia de pulsos periféricos y gangrena. Pocas veces se presenta en la práctica médica diaria, por lo que el diagnóstico es importante para evitar la gangrena. Se presentan tres casos de pacientes atendidos. Se confirmó el diagnóstico clínico y por imágenes, se muestra la evolución y el tratamiento médico realizado.

PALABRAS CLAVE: Tromboflebitis, trombosis de la vena, gangrena. (Fuente: DeCS BIREME).

\section{SUMMARY}

Phlegmasia cerulea dolens (phlebitis, cyanosis, pain) is a rare severe form of deep venous thrombosis, caused by obstruction of the vein flow of the lower limb (usually) with severe cyanosis and circulatory compromise which affects collateral vessels, unlike Phlegmasia alba dolens not compromise collateral vessels. It is characterized by sudden onset of pain, because an increase of the venous pressure, that can lead to pulselessness and gangrene. Is unfrequently in daily medical practice, so the diagnosis is important to avoid gangrene. We present three cases of patients attended with this disease. Diagnosis was confirmed by clinic and imaging; we present the evolution and medical treatment that was performed.

KEYWORDS: Thrombophlebitis, venous thrombosis, gangrene. (Source: MeSH NLM).

\section{INTRODUCCIÓN}

Las complicaciones del tromboembolismo venoso agudo incluyen trombosis venosa profunda (TVP), embolia pulmonar (EP) y síndrome post trombótico que son las causas más comunes de muerte hospitalaria evitable y fuente de morbilidad significativa a largo plazo (1).

Color pálido o cianótico puede ser visto en TVP masiva ocluyendo todas las venas colaterales y finalmente las arterias (2). La pierna pálida es la forma no isquémica o Flegmasia alba dolens, trombosis venosa masiva que aún tienen venas colaterales permeables; cuando hay obstrucción de colaterales es la Flegmasía cerúlea dolens (FCD), la pierna cianótica es isquémica pero aún reversible (3). La isquemia irreversible es la gangrena venosa (GV) que lleva a necrosis (4). La FCD puede ser mal diagnosticada, lo que empeora el pronóstico, evolucionando a gangrena y aumentando la morbimortalidad que es elevada, a pesar del avance de la medicina (5).

En Perú sólo hemos encontrado un artículo publicado acerca FCD y reporta un solo caso (6). Presentamos tres casos de FCD, dos de ellos tratados

\footnotetext{
I Hospital EsSalud Víctor Lazarte Echegaray. Trujillo, Perú

a Cirujano de tórax y cardiovascular.
} 
en el Hospital EsSalud Víctor Lazarte Echegaray y uno de la práctica privada, en la ciudad de Trujillo.

\section{PRESENTACIÓN DE LOS CASOS}

\section{Caso 1}

Mujer de 52 años de edad, casada, nulípara, traída a emergencia del Hospital EsSalud Víctor Lazarte Echegaray, de la ciudad de Trujillo, por dolor de pierna izquierda con cambios de coloración, edema y frialdad; refiriendo un tiempo de enfermedad de cuatro horas. Hicieron la interconsulta a cirugía vascular con la hipótesis diagnóstica de trombosis arterial; había sido operada de fractura de tibia peroné derecha tres semanas antes, por lo que estaba en inmovilización.

Al examen se encontró edema, frialdad, cianosis de pierna izquierda, no se palpaban pulsos poplíteos ni infrapoplíteos (figura 1A). No se pudo realizar eco Doppler vascular de emergencia, pero se realizó angioTEM arterial, comprobándose que las arterias del miembro inferior estaban permeables, sin obstrucciones. El dímero D fue $>7500$ (VN: $<500$ ), se indicó posición Trendelenburg, enoxaparina 60 UI SC bid y anticoagulación oral con warfarina a partir del tercer día.

El eco Doppler venoso realizado al sétimo día del ingreso, no evidenció TVP, salió de alta a los 18 días de hospitalización, con indicación de uso de medias de compresión moderada (figura 1B).

\section{Caso 2}

Mujer de 39 años de edad, casada, obesa, fue traída a emergencia del Hospital EsSalud Víctor Lazarte Echegaray, de la ciudad de Trujillo por sus familiares debido a pérdida de conciencia. Los exámenes auxiliares mostraron: glucosa $354 \mathrm{mg} / \mathrm{dl}$, sodio 149 $\mathrm{mmol} / \mathrm{l}$, urea $60 \mathrm{mg} / \mathrm{dl}$, creatinina $2,3 \mathrm{mg} / \mathrm{dl}$; en orina se encontró glucosa +++ , cuerpos cetónicos +++ , y el análisis de gases arteriales (AGA) mostró, acidosis metabólica.

La paciente llegó en shock; fue intubada y transferida a la unidad de cuidados intensivos, con diagnóstico de coma por diabetes recién diagnosticada. El score APACHE II fue 19; requirió drogas inotrópicas y ventilación mecánica, antibióticoterapia de amplio espectro. SOFA=10 (mortalidad por SOFA: 40-50 \%).

$\mathrm{Al}$ quinto día de ingreso presentó cianosis de mano derecha (figura 2A); ante la sospecha de trombosis arterial le realizaron eco Doppler arterial, no se encontró obstrucción. Se realizó eco Doppler venoso y se diagnosticó trombosis venosa. Recibió enoxaparina, siendo la evolución desfavorable; presentó necrosis de dedos y gangrena venosa al décimo cuarto día de internamiento (figura 2B). Se realizó amputación selectiva. La evolución fue favorable, siendo dada de alta luego de 38 días de hospitalización.
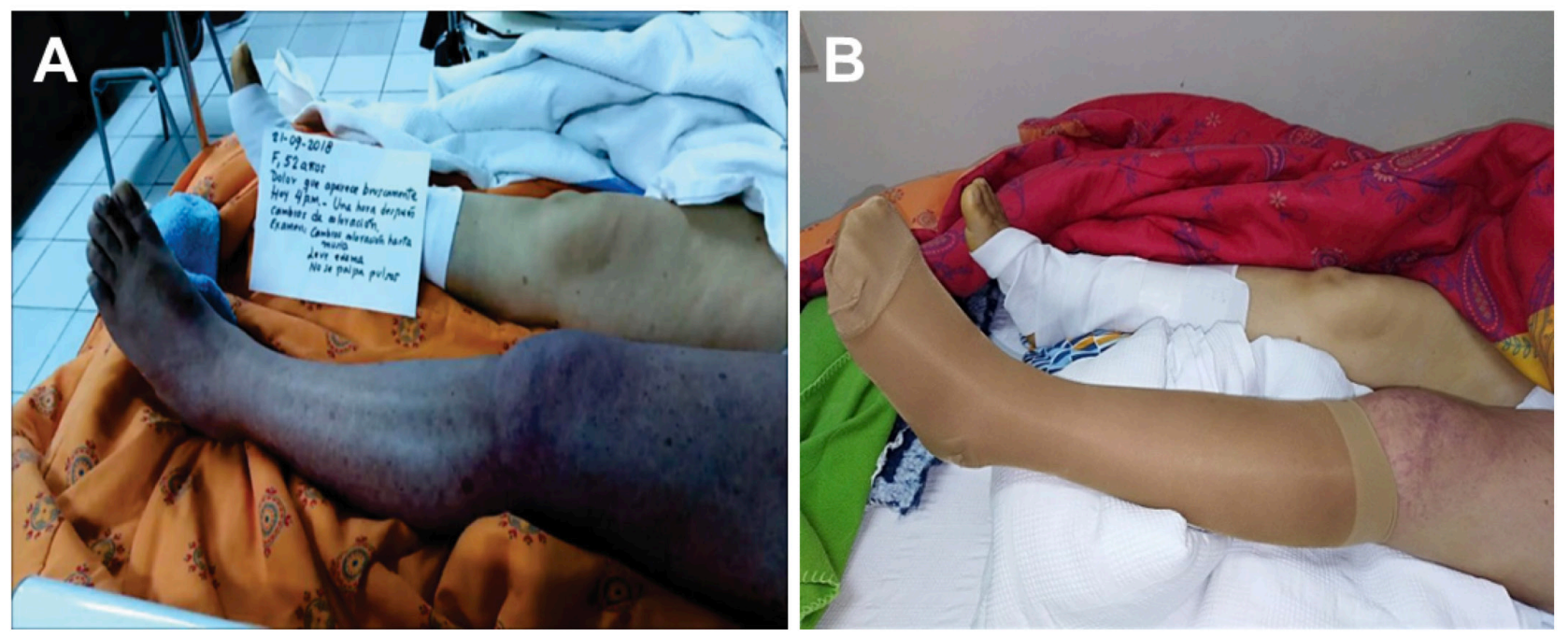

Figura 1. Caso1.A: cianosis severa y edema al ingreso. B: tres días después, sin cianosis, edema resuelto y con medias de compresión. 

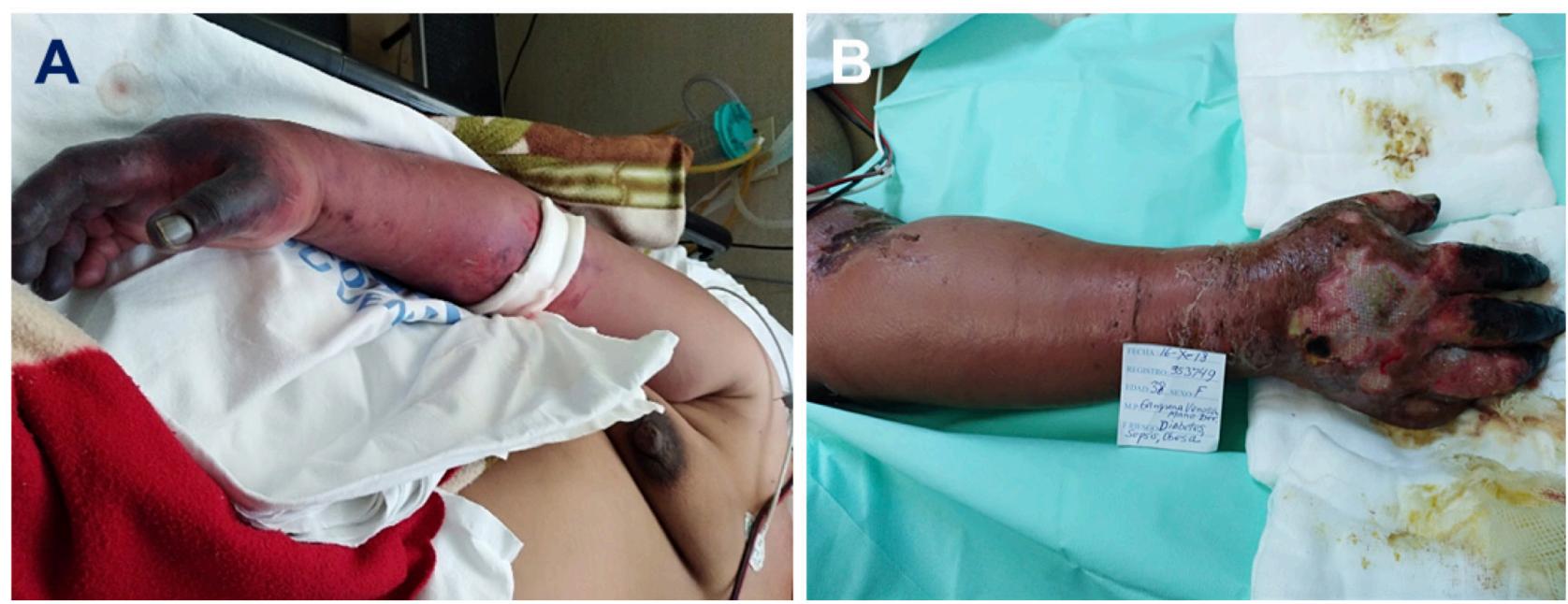

Figura 2. Caso 2. A: Mano izquierda con cianosis al quinto día de ingreso. B: Necrosis de dígitos al 14to día de internamiento.

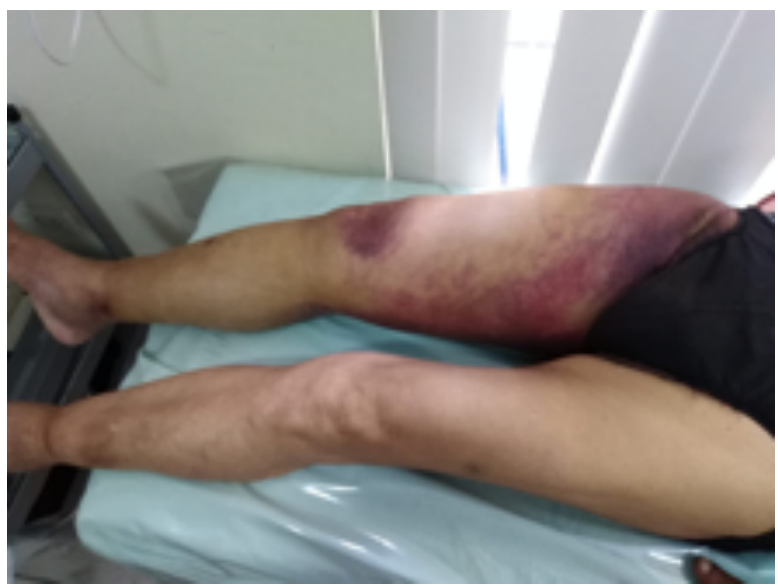

Figura 3. Caso 3. Cianosis de muslo con edema de muslo y pierna.

\section{Caso 3}

Varón de 86 años de edad, procedente de Yungay (Ancash), fue traído a la consulta privada por dolor intenso y edema de pierna derecha; refería un tiempo de enfermedad de cuatro días. El examen demostró, un paciente muy quejumbroso, edema y cianosis de muslo, pulsos ausentes desde la región femoral hasta infrapoplíteos (figura 3). El eco Doppler venoso concluyó: Obstrucción de vena poplítea, femoral superficial y femoral común. Fue internado en una clínica de la ciudad de Trujillo y se indicó posición Trendelenburg y anticoagulación con heparina no fraccionada (HNF).

Los exámenes de laboratorio al ingreso mostraron: hemograma 11200 leucocitos $\mathrm{x} \mathrm{mm^{3 }}$ con abastonados $1 \%$, segmentados $85 \%$, monocitos $5 \%$ y linfocitos $9 \%$; hemoglobina $5,4 \mathrm{~g} / \mathrm{dl}$ anisocitosis, microcitosis y poiquilocitosis; hematocrito $17 \%$, plaquetas 146000

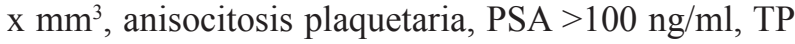
35 segundos, INR 2,37, TPT 67 segundos, Dímero D cuantitativo $>10000$.

La evaluación de urología reveló próstata aumentada de tamaño e irregular; la ecografía hepática mostró tumores hepáticos sugestivos de metástasis. No se podía indicar anticoagulación con warfarina por tener tiempo de protrombina prolongado.

Se continuó la anticoagulación con heparina de bajo peso molecular (HBPM) a las 72 horas de estar con HNF, salió de alta de la clínica luego de siete días de internamiento, con edema y dolor que había disminuido en $70 \%$, con indicación de uso de medias de compresión moderada. Se realizó control en consultorio cinco días después. Se recomendó que se quede en Trujillo unos días más pero el paciente decidió volver a su ciudad natal. No se conoce la evolución posterior.

\section{DISCUSIÓN}

La FCD es una enfermedad rara, se presenta con mayor frecuencia entre la quinta y sexta década de la vida (7), aunque algunos estudios describen entre la sexta y séptima década (8), sin embargo, se ha informado su aparición entre los seis meses y 87 años (4). La edad promedio de los tres casos presentados fue de 59 años que coincidiría con la afirmación de Núñez (7). Su incidencia es mayor en mujeres que en hombres y en miembros inferiores $(7,8)$, los cuadros clínicos pueden sugerir patología arterial aguda $(6,9)$, como ocurrió en dos de los casos presentados; algunos 
piensan que podría ser un efecto adverso por el uso de drogas vasoactivas $(6,7)$, tal como ocurrió en el caso 2 , paciente joven que llegó en shock.

La FCD ocurre cuando aumenta la presión venosa en forma significativa por trombosis masiva del drenaje venoso de la extremidad afectada llevando a trastornos de la perfusión de tejidos, edema, cianosis, frialdad, ausencia de pulsos y culminar en gangrena venosa; tiene un importante impacto en la morbimortalidad, aunque su prevalencia es baja (9).

Los factores de riesgo encontrados en nuestra experiencia son similares a los reportados por otros autores, como es la cirugía reciente (7) en el caso 1; diabetes (3) en el caso 2 y neoplasias $(5,8,9)$ en el caso tres.

El diagnóstico debe ser clínico y confirmado por eco Doppler venoso $(3,4,7,8)$; otros consideran que es mejor el AngioTEM (6,9). En nuestro caso, el diagnóstico clínico fue realizado por el autor de este reporte. En el servicio de emergencia del Hospital Lazarte no se cuenta con equipo de eco Doppler las 24 horas, por lo que, en el caso 1, se realizó AngioTEM para el diagnóstico; en el caso 2 se realizó eco Doppler arterial a solicitud de otro cirujano vascular y posteriormente venoso. En el caso 3, se solicitó solamente eco Doppler venoso.

El tratamiento debe ser inmediato y agresivo, con abordaje multidisciplinario (5), aunque no existe actualmente guía terapéutica consensuada $(8,9)$, los pacientes de EsSalud recibieron heparina de bajo peso molecular (HBPM) y el tercer paciente recibió heparina no fraccionada (HNF). La heparina no fraccionada es sugerida en algunos artículos $(4,5,8)$. Yáñez (6), en Perú, utilizó HBPM en un caso reportado, aunque Brodsky (5), no lo recomienda dado el requerimiento de efecto anticoagulante inmediato.

En los últimos tiempos, se recomienda la instalación de filtro de cava $(3,7,9)$, pero en pacientes con trombocitopenia inducida por heparina con TVP no se debe insertar filtro en cava sino proceder a desobstrucción venosa por cateterismo con trombolisis combinada mecánica y química (5) (no disponible en este momento en Perú). La misma controversia existe entre hacer fasciotomía (6), o no hacerla (5).

Sólo en un artículo se recomendó el uso de pentoxifilina, incluso a largo plazo (4). La trombectomía quirúrgica puede considerarse como último recurso para salvar el miembro amenazado (5). Como complicaciones, en los artículos revisados, se encontró un caso de shock séptico secundario a gangrena venosa que llevó a amputación supracondílea (7) y un paciente que falleció por falla multiorgánica y shock refractario al tercer día de ingreso (9). En los casos que se reporta, no hubo mortalidad, al menos durante la estancia hospitalaria.

En resumen, la FCD es una manifestación grave y poco frecuente de TVP. Es una emergencia médica que con diagnóstico oportuno y un tratamiento precoz se podría salvar el miembro o la vida del paciente. En la realidad nacional, las técnicas endovasculares aún no están a nuestro alcance.

\section{Correspondencia:}

Oswaldo Silva Rodríguez

Avenida Fátima 894-Departamento 102, Víctor Larco, código postal 13009.

Trujillo, Perú.

Correo electrónico: osr_ctcv@yahoo.com

\section{REFERENCIAS BIBLIOGRÁFICAS}

1. Cronenwett J, Johnston W. Rutherford's Vascular Surgery. 8th Edition, Philadelphia: Elsevier; 2014.p. 794.

2. Liapis CD, Balzer K, Benedetti F, Fernandes J. Vascular Surgery. European Manual of Medicine. Berlin: Springer; 2007.

3. Falcón F, Pereda ML, Maripangui M, Campos A, Sarmiento MC. Flegmasia cerulea dolens: reporte de un caso y revisión de la literatura. Rev Hosp Clín Univ Chile. 2010; 21: 124-127. (Citado el 11 de agosto de 2019). Disponible en: https://www. redclinica.cl/Portals/0/Users/014/14/14/ Publicaciones/Revista/flegmasia_cerulea_dolens.pdf

4. Ascaño Ortega, A. Flegmasia cerulea dolens. Rev Cubana Angiol Cir Vasc. 2014; 15(1):00. (Citado el 9 de agosto de 2019). Disponible en: http://scielo.sld.cu/scielo.php?script=sci_arttext\&pid $=$ S1682-00372014000100009

5. Brodsky AL, Melero MJ, Carbia CD. Diagnóstico y tratamiento de la flegmasia cerulea dolens. Hematología. 2009; 13(2):68-72. (Citado el 9 de agosto del 2019). Disponible en: http://www.sah.org. $\mathrm{ar} /$ Revista/numeros/vol13.n2.68-72.pdf

6. Yáñez JE. Flegmasia cerulea dolens. Reporte de caso. Rev Med Hered. 2016; 27(3):168-171. DOI: https:// doi.org/10.20453/rmh.v27i3.2935130X201 6000300008

7. Núñez B, Morales M, Goic I, Carrasco G, Espinoza 
E, González H. Revisión a partir de un caso clínico. Flegmasia cerulea dolens. Rev chil med intensiva. 2009; 24(2): 65-70. (Citado en octubre de 2018) Disponible en: https://www.medicina-intensiva.cl/ revistaweb/revistas/indice/2009-2/4.pdf

8. Barrachina S, García JH, Lorenzo A. Flegmasia cerulea dolens, tratamiento endovascular. Intervencionismo. 2018; 18(3):111-114. (Citado el 13 de agosto de 2019). Disponible en: http://revistaintervencionismo. com/wp-content/uploads/2018_03_casoclinico1.pdf
9. Arnaiz ME, Arnaiz J, Amado CA, García A. Dolor, edema y cianosis: flegmasia cerulea dolens. Med Gen y Fam. 2012; 1(5):250. (Citado el 08 de agosto de 2019). Disponible en: https:/www.medicinaintensiva.cl/revistaweb/revistas/indice/2009-2/4.pdf

Recibido: 19/09/2019

Aceptado: 27/03/2020 